\title{
Spatial and temporal frequency in figure-ground organization
}

\author{
VICTOR KLYMENKO \\ University of North Carolina at Chapel Hill, Chapel Hill, North Carolina \\ NAOMI WEISSTEIN and RICHARD TOPOLSKI \\ State University of New York at Buffalo, Buffalo, New York \\ and \\ CHENG-HONG HSIEH \\ University of North Carolina at Chapel Hill, Chapel Hill, North Carolina
}

\begin{abstract}
Figure-ground organization of an ambiguous pattern can be manipulated by the spatial and temporal frequency content of the two regions of the pattern. Controlling for space-averaged luminance and perceived contrast, we tested patterns in which the two regions of the ambiguous pattern contained sine-wave gratings of $8,4,1$, or 0.5 cycles per degree (cpd) undergoing on:off flicker at the rates of $0,3.75,7.5$, or $15 \mathrm{~Hz}$. For a full set of combinations of temporal frequency differences, with each spatial frequency the higher temporal frequency was seen as background for more of the viewing time. For two spatial frequency combinations, 1 and $4 \mathrm{cpd}$, and 1 and $8 \mathrm{cpd}$, tested under each of the four temporal frequencies, the lower spatial frequency region was seen as the background for more of the viewing time. When the effects of spatial and temporal frequency were set in opposition, neither was predominant in determining perceptual organization. It is suggested that figure-ground organization may parallel the sustained-transient response characteristics of the visual system.
\end{abstract}

The spatiotemporal stimulus flux of the optic array is perceptually organized into objects and events in the visual environment, where figure-ground relationships are assigned between perceptually segregated neighboring regions. This figure-ground separation has been the object of perceptual research since the initial introduction of ambiguous figures by the Gestalt psychologists demonstrated its existence as a unique and distinct visual phenomenon (Koffka, 1935; Rubin 1921/1958). Researchers have investigated the stimulus factors found to influence figure-ground organization (e.g., Farne, 1977; see Uttal, 1981 for a review) and the figure-ground influence on phenomenal appearance (e.g., Coren, 1969). Egusa (1982), for example, found that for two target regions, the target with the greater contrast with its surround will be judged as nearer when it is seen as the figure and as farther when it is seen as the ground. There have, however, been only a few attempts to relate figureground organization to the considerable body of psychophysical data on the spatial and temporal responses of the visual system (Altmann, Eckhorn, \& Singer, 1986; Brown

\footnotetext{
This research was supported by Air Force Office of Scientific Research Grant AFOSR 840115. We thank a reviewer for thoughtful comments and advice. Address reprint requests to Victor Klymenko, Research Division, Radiology Department, University of North Carolina at Chapel Hill, Chapel Hill, NC 27514.
}

\& Weisstein, 1988; Ginsburg, 1978; Klymenko \& Weisstein, 1986; Meyer \& Dougherty, 1987; Schor \& Howarth, 1986). Psychophysical research has tended to focus on visual sensitivity to the spatial and temporal components of stimuli, but little attention has been paid to how higher perceptual processes such as object and depth perception, and figure-ground organization, are influenced by these sensory components (however, see Dodwell \& Caelli, 1984). Recently Klymenko and Weisstein (1986) have reported that the figure-ground organization of ambiguous stationary patterns is influenced by the spatial frequency content of the two regions of the pattern. They found that for a range of spatial frequencies, from 8 to 0.5 cycles per degree (cpd), the region filled with the relatively lower spatial frequency sine-wave grating tends to be seen as the background behind the region filled with the higher spatial frequency grating, which in turn tends to be seen as the figure (see Figure 1). Wong and Weisstein $(1984,1985,1987)$ found that regions of flickering dots tend to be seen as the background behind regions of stationary dots, which in turn are seen as the figure. The transient-sustained continuum of visual response sensitivity (e.g., see Giaschi, Anstis, \& Rogers, 1988; Hess \& Plant, 1985; Kelly, 1972, 1979; Koenderink \& van Doorn, 1979; Robson, 1966; van Nes, Koenderink, \& Bouman, 1967; Watson \& Nachmias, 1977), where visual responses to higher spatial frequencies are generally associated with responses to lower temporal frequencies and 


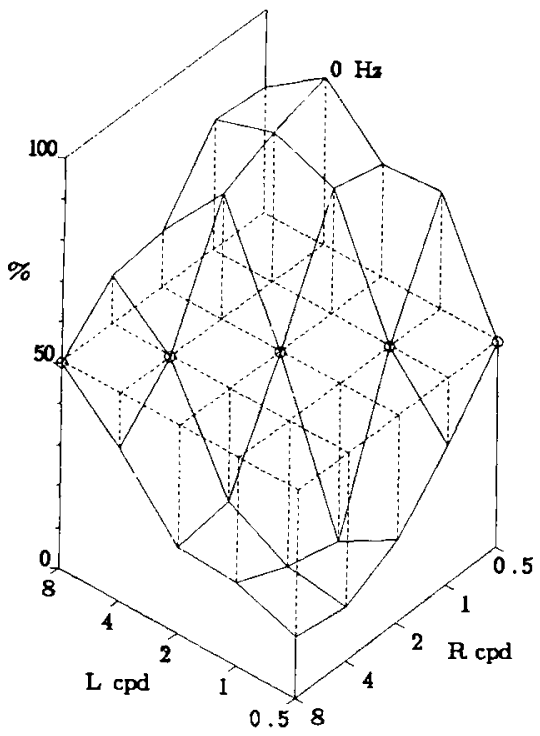

Figure 1. Replotted data from Experiment 2 in Klymenko and Weisstein (1986), where a stationary Maltese crosses pattern, consisting of a right and left region, similar to the pattern used bere, was tested (Hz \# hertz). The coordinates at the base of the graph represent the spatial frequency of the two regions of the ambiguous pattern $(\mathbf{L}$ cpd $=$ spatial frequency of the left region in cycles per degree, $\mathbf{R}$ cpd = spatial frequency of the right region in cycles per degree). The mean percent response times to the $\mathbf{2 0}$ stimuli consisting of all combinations of spatial frequency differences are plotted in terms of height above the base of the graph $(\%=$ mean percent response time the right region was seen as the background). The open circles situated on the dotted grid at the $50 \%$ level represent the hypothetical data where the spatial frequencies of the two regions are equal. For each data point, the distance above or below the $50 \%$ surface is indicated by a vertical dotted line. The data points to the rear of the open circles represent the spatial frequency combinations where the right region contained the relatively lower spatial frequency, and the data points in front of the open circles the higher spatial frequency combinations. In all cases, when the right region contained the lower spatial frequency, it was seen as the ground more than $50 \%$ of the time; when the right region contained the higher frequency, it was seen as the ground less than $50 \%$ of the time.

vice versa, suggests that these response characteristics may play a role in figure-ground organization.

While the differential visual responses to contrasts for different spatial and temporal frequencies (Robson, 1966) become attenuated above threshold (Camisa, Zemon, \& Conte, 1985; Georgeson \& Sullivan, 1975; Kulikowski, 1976), the spatial and temporal frequency content of suprathreshold textures in adjacent regions reliably and systematically determines their figure-ground organization (Klymenko \& Weisstein, 1986; Wong \& Weisstein, $1984,1987)$. As with the early investigations of visual sensitivity to spatial and temporal frequency (see Olzak \& Thomas, 1986 and Watson, 1986 for reviews), the initial investigations of the visual system's responses to figure and ground considered only spatial or temporal frequency separately while holding the other physical dimension constant (Klymenko \& Weisstein, 1986; Wong \& Weisstein, 1984). Below, for suprathreshold stimuli, we systemati- cally examine the combined influence of spatial and temporal frequency on figure-ground organization. We used one of the ambiguous patterns from Klymenko \& Weisstein (1986), the "Maltese crosses" pattern, whose perceptual organization was found to be relatively sensitive to manipulation by spatial frequency differences. In Experiment 1 , we examined the effect of temporal frequency differences between the two regions of the ambiguous pattern where the spatial frequency of the two regions is the same. In Experiment 2, we examined the effect of spatial frequency differences between the two regions where the temporal frequency of the two regions is the same. In Experiment 3, we examined the relative effects of spatial and temporal frequency.

\section{EXPERIMENT 1}

The influence of temporal frequency on perceptual organization was tested for four spatial frequencies.

\section{Method}

\section{Observers}

Fifty-five naive undergraduates, with normal or corrected-tonormal vision, took part in order to fulfill a course requirement. Fourteen, 14, 15, and 12 observers viewed the 8-, 4-, 1- and 0.5cpd spatial frequency patterns, respectively.

\section{Equipment and Stimuli}

A Grinnell GMR-270 image processing system hosted by an LSI$11 / 23$ computer generated the stimuli on a Sony Trinitron KV- 1513 (15-in.) color monitor and recorded the responses, which were made with a joystick. The spatial, temporal, and luminance resolution of the stimuli on the monitor were 64 pixels per degree of visual angle, $30 \mathrm{~Hz}$ (interlaced), and 256 linear luminance levels, respectively. The blue channel was off, and the red and green channels were photometrically equated, producing stimuli varying in brightness from black to bright yellow. The luminance of neutral yellowgrey was $39.0 \mathrm{~cd} / \mathrm{m}^{2}$.

The stimuli consisted of variations of a circular pattern that measured 362 pixels in diameter $\left(5.66^{\circ}\right.$ of visual angle). The circular pattern consisted of eight pie-shaped sectors (see Figure 2). Clockwise from the top, the odd sectors constituted the "right region" and the even sectors constituted the "left region." The borders between the two regions were the horizontal, the vertical, and the two diagonal diameters of the circle. The screen was dark $\left(9.0 \mathrm{~cd} / \mathrm{m}^{2}\right)$ outside of the circle.

For each spatial frequency, the set of stimuli consisted of 12 variations of the basic pattern. The two regions of the circle were each filled with vertical sine-wave gratings $(8,4,1$, or 0.5 cpd), flickering at different rates. Either region could be described as a (stationary or flickering) continuous sine-wave grating that was partially occluded by the other region. Four temporal frequencies were tested: 0 (stationary), $3.75,7.5$, and $15 \mathrm{~Hz}$. For each spatial frequency, each variation consisted of 1 of the 12 combinations of different temporal frequency pairs. The flickering sine-wave gratings underwent on:off flicker; in the "on" half of each temporal cycle the sine-wave grating was present, and in the "off' half of the cycle the region was neutral yellow-grey. The luminance of the sine waves was spatially modulated about neutral yellow-grey. Thus, the space-averaged luminance was constant over time. The contrasts of the gratings were obtained from each observer's contrast matching function. For the patterns in which both regions flickered, the temporal onsets of each of the two gratings were in phase. 


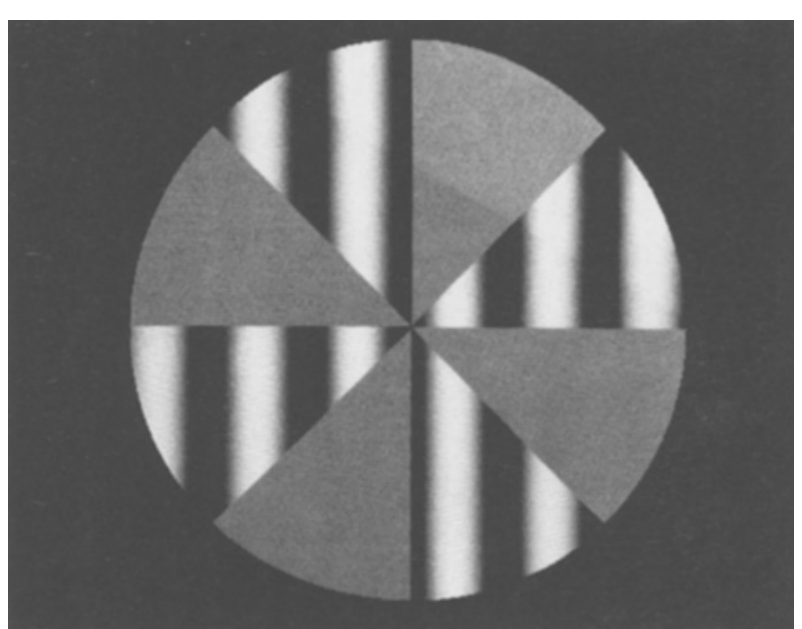

Figure 2. The stimuli in Experiments 1-3 were variations of a circular pattern divided into eight pie-shaped sectors. Each region, consisting of four alternate sectors, contained a sine-wave grating undergoing on:off flicker at one of four rates (see text). Shown is a frame from one of the stimulus sequences used in Experiment 1, where 1-cpd (cycle per degree) flickering sine-wave gratings were tested. The left region is in the "on" portion of its temporal cycle and the right region is in the "off" portion of its cycle.

The stimulus duration was $30 \mathrm{sec}$, and the interval between stimuli was $20 \mathrm{sec}$, during which time the screen was dark.

The stimuli reflect the ecological constraints of the optic array, in that occluding and occluded surfaces and objects tend to be separated by sharp and often abrupt boundaries. In terms of a Fourier description, the spatial and temporal frequency spectrum of the stimuli was more complicated than described here, due both to the sharp boundaries of the patterns and to the spatial, temporal, and luminance resolution of the stimuli as described above. We nominally define spatial frequency in terms of the number of spatial cycles of the sine-wave grating per degree of visual angle, and temporal frequency in terms of the number of on:off cycles of the sine waves per second. The spatial phase of the sine waves was randomly generated to the limit of pixel resolution for each trial.

\section{Procedure}

Observation was monocular with the observer's preferred eye. Each observer sat in a lightproof tunnel in a dark room. A chinrest positioned the observation point $2.19 \mathrm{~m}$ from the display screen.

Contrast matching. Each observer was presented with a series of seven displays. Each display consisted of two vertical sine-wave gratings - the standard grating and one of the test gratings-both with the spatial frequency specific to that group of observers. The contrast of the test grating was controlled by the observer with a joystick. Each grating was contained within a rectangular region, $384 \times 120$ pixels (horizontal $\times$ vertical). Each sine-wave grating started and ended on a neutral yellow-grey portion of the spatial cycle. The centers of the two rectangular regions were 120 pixels above and below the center of the display. The remainder of the display was dark. The lower grating - the standard-was stationary and set to $52 \%$ Michelson contrast. The observers were given unlimited time to observe and test different contrast settings. Each observer matched the perceived contrast of each of the test patterns, which could vary along 23 equal units ranging from $32 \%$ to $81 \%$ contrast, to the standard. The observers first practiced with a test grating that was the same as the standard $(0 \mathrm{~Hz})$. After each observer had met a criterion of three near-perfect matches (plus or minus one unit) in a row, the three test gratings $-3.75,7.5$, and
$15 \mathrm{~Hz}$-were then presented in random order in two blocks. For each observer, the contrasts of the sine waves in the experimental session was $52 \%$ for the $0-\mathrm{Hz}$ gratings, and the average of the two responses for each of the other temporal frequencies. The mean matched contrasts for the four sets of observers are given in Table 1.

Experimental session. After the contrast matching session, a picture of an outline of the circular pattern containing black and white sectors was shown to each observer. The pattern's ambiguity, its two alternative organizations, was described. Each observer was given a randomly generated practice trial. The 12 variations of the pattern were then presented in random order. Each observer was instructed to gaze at the center of the screen at the sound of the warning beep, which preceded stimulus onset by $2 \mathrm{sec}$, and to continue gazing at the center of the pattern for the duration of the stimulus presentation. Each observer was told to respond immediately after the stimulus onset, and to continue responding throughout the trial by moving the joystick to either the right or the left to indicate which of the two regions of the pattern was seen as being "in front" as opposed to in the "background."

\section{Design and Data Analysis}

The six stimuli in which the right region flickered at the higher temporal frequency were designated as the $H i-T F \mathrm{~s}$, and the six stimuli in which the right region flickered at the lower temporal frequency were designated as the $L o-T F$ s. Thus each Hi-TF has a corresponding Lo-TF in which the temporal frequencies of the stimulus pattern's two regions were reversed.

The response measure was the percentage of the total response time during which the observers saw the right region as the background, where the total response time was the total amount of time during which observers responded either right or left (neutral responses were discounted). ${ }^{1}$ The percent times for each spatial frequency were analyzed by a one-way repeated measures analysis of variance (ANOVA) with 12 treatments and six planned contrasts, which compared each Hi-TF with its corresponding Lo-TF.

In addition, the percent times were tested for a linear trend (Robson, 1959) with respect to the magnitude of the temporal frequency difference between the two regions of the pattern. For example, the temporal frequency of the right region minus the temporal frequency of the left region in the $0 \times 3.75 \mathrm{~Hz} \mathrm{Hi}-\mathrm{TF}$ is $3.75 \mathrm{~Hz}$. If we consider this difference as one positive unit, then on a linear scale the $0 \times 15 \mathrm{~Hz} \mathrm{Hi}$-TF will be +4 units, the $15 \times 0 \mathrm{~Hz}$ LoTF will be -4 units, and the other 10 treatments will fall between the two extremes.

\section{Results}

The percentage of the response time during which the right region was seen as the background was the measure of figure-ground organization. The right region flickered at the relatively higher temporal frequency in the $\mathrm{Hi}-$ TFs, and at the lower temporal frequency in the Lo-TFs.

Table 1

Mean Matched Contrasts and Standard Deviations for Experiment 1

\begin{tabular}{llrlllll}
\hline \multirow{2}{*}{$\begin{array}{c}\text { Spatial } \\
\text { Frequency }\end{array}$} & \multicolumn{6}{c}{ Temporal Frequency (Hz) } \\
\cline { 2 - 8 } (cpd) & \multicolumn{2}{c}{3.75} & & \multicolumn{2}{c}{7.5} & & 15 \\
\hline 8 & 62 & 10 & 61 & 9 & 71 & 6 \\
4 & 52 & 5 & 51 & 5 & 56 & 9 \\
1 & 47 & 3 & 43 & 3 & 47 & 5 \\
0.5 & 49 & 3 & 47 & 3 & 50 & 6 \\
\hline
\end{tabular}

Note-Means represent the average percent contrasts for each test sinewave grating obtained from matching to a stationary $52 \%$ contrast grating of the same spatial frequency. 
Table 2

Mean Percent Response Times for Experiment 1

\begin{tabular}{cccccc}
\hline $\begin{array}{c}\text { Spatial } \\
\text { Frequency } \\
\text { (cpd) }\end{array}$ & $\begin{array}{c}\text { Temporal } \\
\text { Frequency } \\
\text { of Left } \\
\text { Region (Hz) }\end{array}$ & \multicolumn{4}{c}{$\begin{array}{c}\text { Temporal Frequency } \\
\text { of Right Region (Hz) }\end{array}$} \\
\hline 8 & 0 & 0 & 3.75 & 7.5 & 15 \\
\hline 8 & 3.75 & 31.1 & - & 46.8 & 63.9 \\
& 7.5 & 24.6 & 58.9 & - & 50.7 \\
& 15 & 12.6 & 47.1 & 47.0 & - \\
4 & 0 & - & 63.6 & 60.6 & 65.3 \\
& 3.75 & 26.1 & - & 54.4 & 72.6 \\
& 7.5 & 26.4 & 47.9 & - & 63.4 \\
& 15 & 21.2 & 35.1 & 43.7 & - \\
1 & 0 & - & 50.9 & 82.1 & 77.7 \\
& 3.75 & 27.0 & - & 66.1 & 58.7 \\
& 7.5 & 14.1 & 34.9 & - & 62.5 \\
& 15 & 15.6 & 56.3 & 46.4 & - \\
0.5 & 0 & - & 72.9 & 59.2 & 67.5 \\
& 3.75 & 20.1 & - & 60.3 & 32.4 \\
& 7.5 & 33.4 & 59.3 & - & 47.8 \\
& 15 & 48.3 & 31.3 & 31.1 & - \\
\hline
\end{tabular}

Note-Mean percent response times represent the percentage of the response time that the right region was seen as the background. Above each of the the blank diagonals are the Hi-TFs, those stimuli where the right region contained the relatively higher temporal frequency sinewave grating, and below the diagonals are the Lo-TFs, those stimuli where the right region contained the lower temporal frequency grating.

The effect of stimulus variation was significant for each of the four spatial frequencies: for $8 \mathrm{cpd}, F(11,143)=$ $7.58, M S_{\mathrm{r}}=4.74, p<.01$; for $4 \mathrm{cpd}, F(11,143)=$ $5.79, M S_{\mathrm{r}}=3.84, p<.01$; for $1 \mathrm{cpd}, F(11,154)=$ $9.48, M S_{\mathrm{r}}=3.56, p<.01$; and for $0.5 \mathrm{cpd}, F(11,121)$ $=2.30, M S_{\mathrm{r}}=10.52, p<.05$. Table 2 shows the mean percent times that the right region was seen as the background for each of the temporal frequency variations. Collectively, for all four spatial frequencies, 21 out of the $24 \mathrm{Hi}-\mathrm{TFs}$ had a mean percent time above $50 \%$, and 21 out of the $24 \mathrm{~L} o$-TFs had a mean percent time below $50 \%$, which indicates that overall the relatively higher temporal frequency region was seen as the background more of the time. The percent times shown in Table 2 are graphed in Figure 3.

For each spatial frequency there were six planned comparisons, to compare each $\mathrm{Hi}-\mathrm{TF}$ with its corresponding Lo-TF. This is the difference in height between corresponding points on opposite sides of the open circles on the response surfaces shown in Figure 3. The results of the planned comparisons for each temporal frequency combination are shown in Table 3 . For all four spatial frequencies considered together, 23 out of 24 of the $\mathrm{Hi}$ TFs are greater than the corresponding Lo-TFs, indicating that the higher temporal frequency region is perceptually biased towards being seen as the background: specifically, this means that when the right region contains the relatively higher temporal frequency grating, it is seen as background more of the time than when it contains the lower frequency grating. Only one of the planned comparisons was significant for the lowest spatial frequency, indicating that the figure-ground system may be more sensitive to temporal frequency differences for higher spatial frequencies.

The tests for a linear trend of percent time with respect to the magnitude of the temporal frequency difference between the two regions of each pattern was significant for each of the four spatial frequencies: for $8 \mathrm{cpd}, F(1,143)$ $=52.25, p<.01$; for $4 \mathrm{cpd}, F(1,143)=51.50$, $p<.01$; for $1 \mathrm{cpd}, F(1,154)=61.36, p<.01$; and for $0.5 \mathrm{cpd}, F(1,121)=5.43, p<.05$. These results support the general observation that the greater the difference in temporal frequency, the greater the amount of viewing time that the higher temporal frequency region will be seen as the background.

\section{EXPERIMENT 2}

The perceptual organizations of two spatial frequency pairs were examined under different temporal conditions.

\section{Method}

Except for the differences described below, the method was the same as that in Experiment 1.

\section{Observers}

Twelve new observers viewed the 1- and 4-cpd combination, and another 12 new observers viewed the 1- and 8-cpd combination.

\section{Equipment and Stimuli}

The two regions of the circle were filled with sine-wave gratings differing in spatial frequency: 1 and 4 cpd for the first set of observers, and 1 and $8 \mathrm{cpd}$ for the second set. For each spatial frequency combination, the set of stimuli consisted of 8 variations of the basic pattern. The right region contained the lower spatial frequency grating in four of the variations and the higher frequency grating in the other four. The entire pattern flickered at one of three rates- $3.75,7.5,15 \mathrm{~Hz}-$ or it was stationary $(0 \mathrm{~Hz})$. In the flickering patterns, when one region was in the "on" half of its temporal cycle, the other region was in the "off" half of its temporal cycle, and vice versa. The interval between stimuli was $8 \mathrm{sec}$.

\section{Procedure}

Contrast matching. The standard was a $2-\mathrm{cpd}, 0-\mathrm{Hz}$ sine-wave grating set to $52 \%$ contrast. The mean percent contrasts for each of the test gratings obtained from matching to the standard are given in Table 4 for both sets of observers.

\section{Design and Data Analysis}

The four variations in which the right region contained the lower spatial frequency grating are designated as the $L o-S F s$, and the four variations in which the right region contains the higher spatial frequency are designated as the Hi-SFs. The percent times for each spatial frequency combination were analyzed by a one-way repeated measures ANOVA with 8 treatments and 4 planned contrasts, which compared each Lo-SF with its corresponding Hi-SF, where the spatial frequencies were reversed.

\section{Results}

The time during which the right region was seen as the background was the measure of figure-ground organization. The effect of stimulus variation was significant for both spatial frequency combinations: for 1 and $4 \mathrm{cpd}$, 

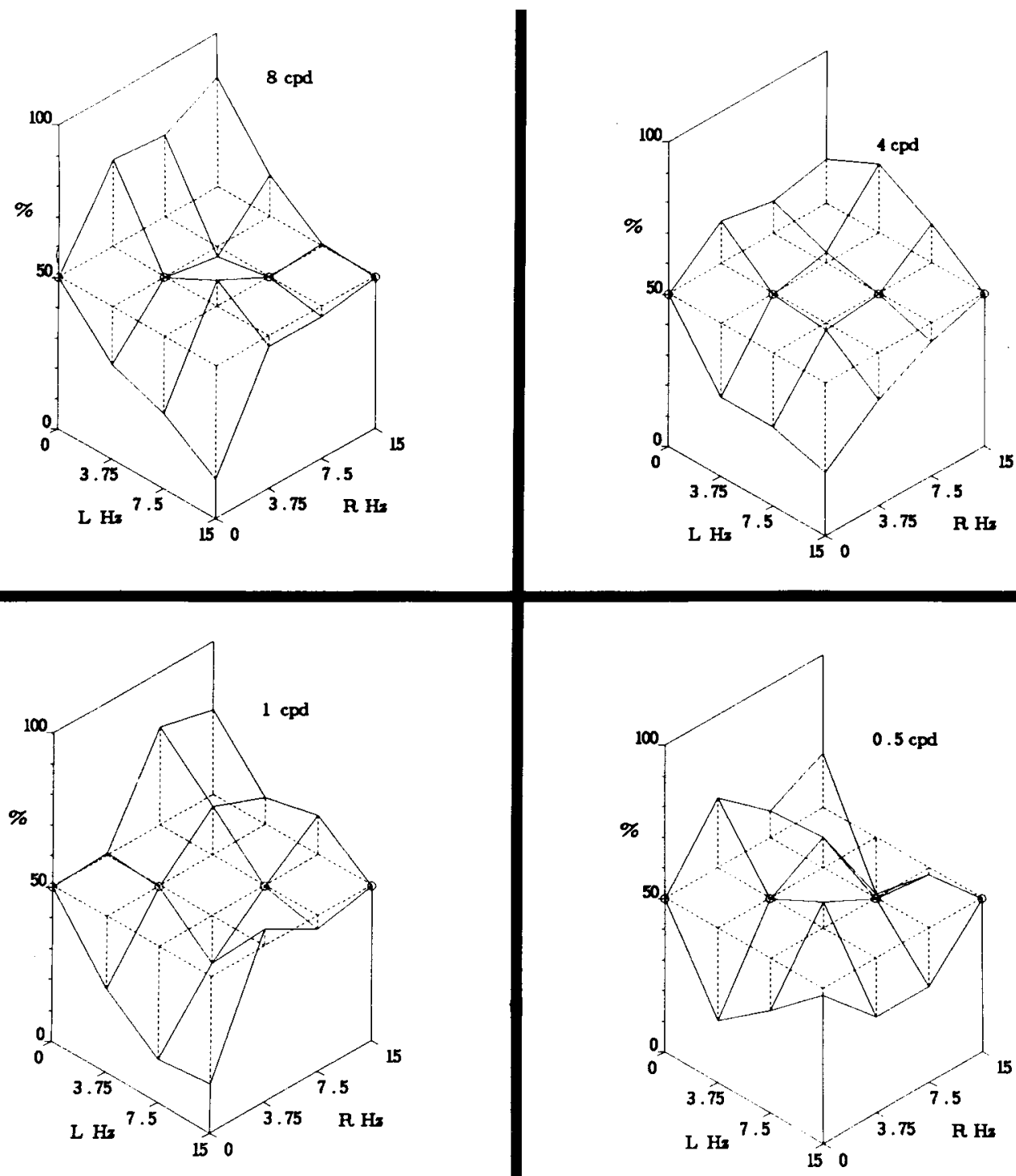

Figure 3. The temporal frequency response surfaces for Experiment 1 for each spatial frequency (cpd). The numerical data in Table 2 are plotted in terms of height above the base of the graph (\% = mean percent response time the right region was seen as background). The coordinates at the base of each graph represent the temporal frequency of the two regions ( $\mathrm{L} \mathrm{Hz}=$ temporal frequency of left region in hertz; $\mathbf{R ~ H z}=$ temporal frequency of right region in hertz). The four open circles situated on the dotted grid located at the $50 \%$ level represent the blank diagonals in Table 2 , where the temporal frequencies of the two regions are equal. For each data point, the distance above or below the $50 \%$ surface is indicated by a vertical dotted line. The data points to the rear of the open circles represent the Hi-TFs, where the right region contained the relatively higher temporal frequency grating, and the data points in front of the open circles represent the Lo-TFs, where the right region contained the lower frequency grating.

$F(7,77)=6.12, M S_{\mathrm{r}}=4.45, p<.01 ;$ for 1 and $8 \mathrm{cpd}$, $F(7,77)=6.67, M S_{\mathrm{r}}=8.64, p<.01$. Table 5 shows the mean percent times during which the right region was seen as the background for each temporal condition. All of the Lo-SFs had mean percent times above $50 \%$ and were larger than the corresponding Hi-SFs, all of which had mean percent times below $50 \%$. The larger values for Lo-SFs than for Hi-SFs indicate that the lower spatial frequency region is perceptually biased towards being seen as the background; specifically, this indicates that when the right region contains the relatively lower spatial frequency grating, it is seen as background more of the time than when it contains the higher frequency grating. The results of the planned contrasts, which compared each Lo-SF with its corresponding Hi-SF, are shown in the right column of Table 5. Each of these differences was significant, but for one exception: the $15-\mathrm{Hz}$ percent time for the 1- and 4-cpd combination, which only marginally failed to reach significance. For both spatial frequency combinations, the largest difference was in the sta- 
Table 3

Results of the Planned Comparisons for Experiment 1

\begin{tabular}{cccccccc}
\hline \multirow{2}{*}{$\begin{array}{c}\text { Spatial } \\
\text { Frequency } \\
\text { (cpd) }\end{array}$} & $\begin{array}{c}\text { Planned } \\
\text { Comparison } \\
\text { Test }\end{array}$ & \multicolumn{6}{c}{ Temporal Frequency Combinations (Hz) } \\
\cline { 3 - 8 } & $0 \& 3.75$ & $0 \& 7.5$ & $0 \& 15$ & $3.75 \& 7.5$ & $3.75 \& 15$ & $7.5 \& 15$ \\
\hline 8 & $F(1,143)$ & $17.29 \dagger$ & $20.98 \dagger$ & $40.59 \dagger$ & 1.11 & 2.12 & 0.10 \\
4 & $F(1,143)$ & $13.08 \dagger$ & $10.85 \dagger$ & $18.07 \dagger$ & 0.39 & $13.03 \dagger$ & 3.59 \\
1 & $F(1,154)$ & $5.33^{*}$ & $43.27 \dagger$ & $36.13 \dagger$ & $9.11 \dagger$ & 0.05 & 2.44 \\
0.5 & $F(1,121)$ & $11.05 \dagger$ & 2.63 & 1.47 & 0.01 & 0.01 & 1.10 \\
\hline${ }^{*} p<.05$. & $\dagger p<.01$. & & & & & &
\end{tabular}

tionary condition, indicating that flicker may attenuate the effect of spatial frequency differences.

\section{EXPERIMENT 3}

The combined results for Experiments 1 and 2 suggest that if one of the regions of the ambiguous pattern has both a lower spatial frequency and a higher temporal frequency, then both factors should bias this region to be perceived as the background. Informal observation indicates that this is clearly the case. However, what if the

Table 4

Mean Matched Contrasts and Standard Deviations for Experiment 2

\begin{tabular}{|c|c|c|c|c|c|c|c|c|}
\hline \multirow{3}{*}{$\begin{array}{l}\text { Spatial } \\
\text { Frequency } \\
\text { (cpd) }\end{array}$} & \multicolumn{8}{|c|}{ Temporal Frequency (Hz) } \\
\hline & \multicolumn{2}{|c|}{0} & \multicolumn{2}{|c|}{3.75} & \multicolumn{2}{|c|}{7.5} & \multicolumn{2}{|c|}{15} \\
\hline & $M$ & $\overline{S D}$ & $\bar{M}$ & $\overline{S D}$ & $\bar{M}$ & $S D$ & $\bar{M}$ & $\widehat{S D}$ \\
\hline 1 & 62 & 7 & 55 & 6 & 52 & 4 & 54 & 4 \\
\hline 4 & 47 & 5 & 50 & 7 & 45 & 7 & 51 & 12 \\
\hline 1 & 60 & 5 & 53 & 5 & 51 & 7 & 58 & 7 \\
\hline 8 & 47 & 8 & 52 & 12 & 50 & 13 & 59 & 13 \\
\hline
\end{tabular}

Note-Means represent the average percent contrasts for each test sinewave grating obtained from matching to a stationary 2 -cpd grating set at $52 \%$ contrast. The top two rows are from the 1 - and 4 -cpd observers and the bottom two rows are from the 1- and 8-cpd observers.

Table 5

Mean Percent Response Times and Results of the Planned Comparisons for Experiment 2

\begin{tabular}{|c|c|c|c|c|}
\hline \multirow{2}{*}{$\begin{array}{c}\text { Spatial } \\
\text { Frequency } \\
\text { Combination } \\
\text { (cpd) }\end{array}$} & \multirow{2}{*}{$\begin{array}{c}\text { Temporal } \\
\text { Frequency } \\
(\mathbf{H z})\end{array}$} & \multicolumn{2}{|c|}{$\begin{array}{c}\text { Mean Percent } \\
\text { Response Times }\end{array}$} & \multirow{2}{*}{$\begin{array}{c}\text { Planned } \\
\text { Comparison } \\
\text { Test } \\
\end{array}$} \\
\hline & & Lo-SFs & Hi-SFs & \\
\hline \multirow{5}{*}{1 and 4} & & & & $F(1,77)$ \\
\hline & 0 & 75.7 & 31.81 & $18.00 \dagger$ \\
\hline & 3.75 & 59.4 & 30.0 & $8.11 \dagger$ \\
\hline & 7.5 & 64.6 & 30.7 & $10.78 \dagger$ \\
\hline & 15 & 63.2 & 42.9 & 3.84 \\
\hline \multirow[t]{5}{*}{1 and 8} & & & & $F(1,77)$ \\
\hline & 0 & 70.3 & 13.5 & $15.58 \dagger$ \\
\hline & 3.75 & 67.5 & 21.1 & $10.39 \dagger$ \\
\hline & 7.5 & 58.0 & 25.5 & $5.10^{*}$ \\
\hline & 15 & 64.8 & 9.0 & $15.04 \dagger$ \\
\hline
\end{tabular}

Note-Mean percent response times represent the percentage of the response time that the right region was seen as the background. The Lo-SFs are the stimuli where the right region contained the relatively lower spatial frequency sine-wave grating, and the Hi-SFs are the stimuli where the right region contained the higher frequency grating. ${ }^{*} p<.05$. $t_{p}<.01$. effects of spatial frequency and temporal frequency are set in opposition? The perceptual organizations of the same two spatial frequency pairs tested in Experiment 2 were examined under this condition.

\section{Method}

Except for the differences described below, the method was the same as that in Experiment 2.

\section{Observers}

Twelve and 11 new observers, respectively, viewed the spatial frequency combinations 1 and $4 \mathrm{cpd}$ and 1 and $8 \mathrm{cpd}$.

\section{Equipment and Stimuli}

For each spatial frequency combination, the set of stimuli consisted of 12 variations of the basic pattern. The right region contained the lower spatial frequency in six of the variations and the higher spatial frequency in the other six. Four temporal frequencies were tested: $0,3.75,7.5$, and $15 \mathrm{~Hz}$. All combinations of temporal frequency differences were tested, with the following qualification. The higher spatial frequency grating always flickered at the higher temporal frequency.

\section{Procedure}

Contrast matching. For both sets of observers, the average percent contrasts obtained from matching to the standard, a 2-cpd 0 $\mathrm{Hz}$ grating set at $52 \%$ contrast, are given in Table 6.

\section{Design and Data Analysis}

For 6 of the 12 patterns, designated the Hi-TF Hi-SFs, the right region contained both the higher spatial frequency and the higher temporal frequency grating. Thus the spatial frequency would tend to make the right region the figure, whereas the temporal frequency would tend to make it the ground. For the other 6 patterns, designated as the Lo-TF Lo-SFs, the right region contained both the lower spatial and the lower temporal frequency, where each stimulus dimension would correspondingly tend to induce the reverse. The percent times for each spatial frequency combination were analyzed by a one-way repeated measures ANOVA with 12 treatments and six planned contrasts, which compared each Hi-TF Hi-SF with its corresponding Lo-TF Lo-SF.

\section{Results}

The time during which the right region was seen as the background was the measure of figure-ground organization. The mean percent times are given in Table 7. The effect of stimulus variation was not significant for either spatial frequency combination: for 1 and $4 \mathrm{cpd}, F(11,121)$ $=1.18, M S_{\mathrm{r}}=3.15$; for 1 and $8 \mathrm{cpd}, F(11,110)=0.93$, $M S_{\mathrm{r}}=13.47$. The results of the planned comparisons, which compared each Hi-TF Hi-SF with its corresponding Lo-TF Lo-SF, are shown in Table 8. Only one of 
Table 6

Mean Matched Contrasts and Standard Deviations for Experiment 3

\begin{tabular}{|c|c|c|c|c|c|c|c|c|}
\hline \multirow{3}{*}{$\begin{array}{c}\text { Spatial } \\
\text { Frequency } \\
\text { (cpd) }\end{array}$} & \multicolumn{8}{|c|}{ Temporal Frequency $(\mathrm{Hz})$} \\
\hline & \multicolumn{2}{|c|}{0} & \multicolumn{2}{|c|}{3.75} & \multicolumn{2}{|c|}{7.5} & \multicolumn{2}{|c|}{15} \\
\hline & $M$ & $S D$ & $M$ & $S D$ & $M$ & $S D$ & $M$ & $S D$ \\
\hline 1 & 61 & 6 & 52 & 6 & 51 & 5 & 54 & \\
\hline 4 & 43 & 6 & 45 & 6 & 43 & 6 & 48 & \\
\hline 1 & 63 & 8 & 58 & 6 & 52 & 6 & 58 & \\
\hline 8 & 50 & 11 & 56 & 12 & 54 & 8 & 62 & \\
\hline
\end{tabular}

Note-Means represent the average percent contrasts for each test sinewave grating obtained from matching to a stationary 2 -cpd grating set at $52 \%$ contrast. The top two rows are from the 1- and 4-cpd observers, and the bottom two rows are from the 1- and 8-cpd observers.

Table 7

Mean Percent Response Times for Experiment 3

\begin{tabular}{|c|c|c|c|c|c|}
\hline \multirow{2}{*}{$\begin{array}{c}\text { Spatial } \\
\text { Frequency } \\
\text { Combination } \\
\text { (cpd) }\end{array}$} & \multirow{2}{*}{$\begin{array}{c}\text { Temporal } \\
\text { Frequency } \\
\text { of Left } \\
\text { Region }(\mathrm{Hz}) \\
\end{array}$} & \multicolumn{4}{|c|}{$\begin{array}{l}\text { Temporal Frequency } \\
\text { of Right Region }(\mathrm{Hz})\end{array}$} \\
\hline & & 0 & 3.75 & 7.5 & 15 \\
\hline 1 and 4 & $\begin{array}{l}0 \\
3.75 \\
7.5 \\
15\end{array}$ & $\begin{array}{l}- \\
47.8 \\
34.3 \\
38.4\end{array}$ & $\begin{array}{l}51.0 \\
- \\
53.6 \\
56.0\end{array}$ & $\begin{array}{c}53.7 \\
44.4 \\
- \\
49.3\end{array}$ & $\begin{array}{c}54.8 \\
44.8 \\
48.8 \\
-\end{array}$ \\
\hline 1 and 8 & $\begin{array}{l}0 \\
3.75 \\
7.5 \\
15\end{array}$ & $\begin{array}{l}- \\
26.0 \\
38.5 \\
50.4\end{array}$ & $\begin{array}{c}47.5 \\
- \\
62.7 \\
62.0\end{array}$ & $\begin{array}{c}46.0 \\
52.3 \\
- \\
58.8\end{array}$ & $\begin{array}{c}67.1 \\
47.9 \\
40.4 \\
- \\
\end{array}$ \\
\hline
\end{tabular}

Note-Mean percent response times represent the percentage of the response time that the right region was seen as the background. Above each blank diagonal are the Hi-TF Hi-SFs, the stimuli where the right region contained both the higher temporal frequency and the higher spatial frequency sine-wave grating, and below the diagonals are the Lo-TF Lo-SFs, the stimuli where the right region contained both the lower temporal frequency and the lower spatial frequency grating.

these reached significance; for the 1- and 4-cpd combination, the right region in the $0-$ and $7.5-\mathrm{Hz} \mathrm{Hi-TF} \mathrm{Hi}-$ $\mathrm{SF}$ variation was seen as the ground more of the time than was the right region in the corresponding Lo-TF Lo-SF. This indicates that for this stimulus combination, the effect of temporal frequency overcame the effect of spatial frequency.

\section{DISCUSSION}

Visual mechanisms are differentially sensitive to different spatiotemporal cross sections of the optic array, in terms of spatial and temporal frequency (Robson, 1966; see also Livingston \& Hubel, 1988). At threshold, the response to the stimulus is mediated by the most sensitive mechanism. The findings of Wong and Weisstein $(1982$, 1983) suggest a functional relationship between figure-ground perception and the transient and sustained responses of the visual system. The relatively more sustained mechanisms are relatively more sensitive to higher spatial and lower temporal frequencies and are thought to signal "figure" with respect to the more transient mechanisms, which are more sensitive to the lower spatial and higher temporal frequencies and are thought to signal "'ground." Considered in terms of ecological utility, it has been argued (e.g., by Calis \& Leeuwenberg, 1981) that the ground system, which is faster, acts as an early warning system signaling global information and motion and thus regions of potential interest, whereas the figure system is specialized for finer spatial resolution and detailed analysis of the region of interest itself (see discussions in Breitmeyer, 1980, 1984; Breitmeyer \& Ganz, 1976; Julesz, 1978; and Weisstein \& Wong, 1986, 1987). For example, low spatial frequency stimuli have more optokinetic potential than high spatial frequency stimuli (see discussion in Wertheim, in press). We have found systematic effects of the spatial and temporal frequency of suprathreshold stimuli on figure-ground organization. Experiment 2 extends to flickering patterns Klymenko and Weisstein's (1986) results for stationary patterns (see Figure 1), in that the relatively lower spatial frequency region tends to be seen as the background behind the higher spatial frequency region; this figure-ground separation is attenuated for some temporal frequencies, presumably because there is less differential stimulation of the transient and sustained mechanisms by the two regions when both are flickering. Wong and Weisstein $(1984,1987)$ found the maximal grounding of the flickering region behind a stationary region to occur for flicker rates around 6 to $8 \mathrm{~Hz}$, with a drop-off in the "flickerinduced ground effect" at lower and higher temporal frequencies. We did not find any consistent tuning of the flicker-induced ground effect with respect to temporal frequency. In Experiment 1, we found that at up to $15 \mathrm{~Hz}$ the higher temporal frequency region tends to be seen as the background more of the time, with this effect more pronounced for higher spatial frequencies. Wong and Weisstein $(1984,1987)$, have used random-dot stimuli that contain multiple spatial frequency components, and controlled for time-averaged luminance, while we have used relatively low spatial frequency sine-wave stimuli, and controlled for space-averaged luminance and perceived

Table 8

Results of the Planned Comparisons for Experiment 3

\begin{tabular}{|c|c|c|c|c|c|c|c|}
\hline \multirow{2}{*}{$\begin{array}{c}\text { Spatial } \\
\text { Frequency } \\
\text { Combination } \\
\text { (cpd) }\end{array}$} & \multirow{2}{*}{$\begin{array}{c}\text { Planned } \\
\text { Comparison } \\
\text { Test } \\
\end{array}$} & \multicolumn{6}{|c|}{ Temporal Frequency Combinations $(\mathrm{Hz})$} \\
\hline & & $0 \& 3.75$ & $0 \& 7.5$ & $0 \& 15$ & $3.75 \& 7.5$ & $3.75 \& 15$ & $7.5 \& 15$ \\
\hline $\begin{array}{l}1 \text { and } 4 \\
1 \text { and } 8\end{array}$ & $\begin{array}{l}F(1,121) \\
F(1,110)\end{array}$ & $\begin{array}{l}0.13 \\
1.55\end{array}$ & $\begin{array}{l}4.99 * \\
0.19\end{array}$ & $\begin{array}{l}3.56 \\
0.94\end{array}$ & $\begin{array}{l}1.11 \\
0.37\end{array}$ & $\begin{array}{l}1.65 \\
0.67\end{array}$ & $\begin{array}{l}0.003 \\
1.15\end{array}$ \\
\hline
\end{tabular}


contrast of the flickering pattern. The relative influence of these factors is a question for further research.

The spatial frequency results are counterintuitive, in that one might have expected the larger-patterned low spatial frequency gratings to appear as the figure in front of the smaller-patterned high spatial frequency gratings, as predicted by size-distance constancy (see discussion in Klymenko \& Weisstein, 1986). On the other hand, there are countless real-world situations consistent with the pattern of temporal frequency results. For example, consider an observer who visually pursues a small, laterally moving object in a textured environment (see Hochberg, 1971): more background than foreground texture elements will sweep over the retina, producing a higher rate of transients for the background than the foreground. In addition, transients are produced by the occlusion and disocclusion of background texture by the moving object (see Gibson, Kaplan, Reynolds, \& Wheeler, 1969; see also Ramachandran \& Anstis, 1986); these transients may specify the figure and ground regions. The ecological interpretation of these data is still an open question.

In Experiment 1, where the spatial frequency was held constant, the relatively higher temporal frequency region tended to be seen as the ground. In Experiment 2, where spatial frequency differences were tested under different temporal frequencies, the relatively lower spatial frequency region tended to be seen as the ground. The sustained-transient hypothesis of figure-ground organization predicts a tradeoff in the effects of spatial and temporal frequency. This tradeoff is evident in Experiment 3, where temporal and spatial frequencies were set in opposition. Regions with relatively low spatial frequency and low temporal frequency were seen as ground as often as were regions with relatively high spatial frequency and high temporal frequency. Neither region stimulated the sustained (figure) mechanisms, or the transient (ground) mechanisms better than the other. These data support the notion that the transient and sustained mechanisms of the visual system are functionally related to the figure-ground organization of visual space-time.

\section{REFERENCES}

Altmann, L., Eckhorn, R., \& Singer, W. (1986). Temporal integration in the visual system: Influence of temporal dispersion on figureground discrimination. Vision Research, 26, 1949-1957.

BREITMEYER, B. G. (1980). Unmasking visual masking: A look at the "why" behind the veil of the "how." Psychological Review, 87, 52-69.

BREITMEYER, B. G. (1984). Visual masking: An integrative approach. New York: Oxford University Press.

BREITME yer, B. G., GANZ, L. (1976). Implications of sustained and transient channels for theories of visual pattern masking, saccadic suppression, and information processing. Psychological Review, 83, 1-36.

Brown, J. M., WEISstein, N. (1988). A spatial frequency effect on perceived depth. Perception \& Psychophysics, 44, 157-166.

Calis, G., LeEuwenberg, E. (1981). Grounding the figure. Journal of Experimental Psychology: Human Perception \& Performance, 7, 1386-1397.

Camisa, J., Zemon, V., Conte, M. (1985). Contrast matching and temporal frequency. Supplement to Investigative Ophthalmology \& Visual Science, 26(3), 138. (Abstract)
Coren, S. (1969). Brightness contrast as a function of figure-ground relations. Joumal of Experimental Psychology, 80, 517-524.

DodWell, P. C., \& CAELu, T. (Eds.) (1984). Figural synthesis. Hillsdale, NJ: Erlbaum.

EGUSA, H. (1982). Effect of brightness on perceived distance as a figureground phenomenon. Perception, 11, 671-676.

FARNE, M. (1977). Brightness as an indicator to distance: Relative brightness per se or contrast with the background? Perception, 6, 287-293.

Georgeson, M. A., Sullivan, G. D. (1975). Contrast constancy: Deblurring in human vision by spatial frequency channels. Journal of Physiology, 256, 627-656.

Giaschi, D., ANSTIS, S., \& Rogers, B. (1988). Adaptation to spatially uniform flicker raises the apparent spatial frequency of low-frequency gratings. Investigative Ophthalmology \& Visual Science, 29, 370. (Abstract)

Gibson, J. J., Kaplan, G. A., Reynolds, H. N., JR., \& Wheeler, K. (1969). The change from visible to invisible: A study of optical transitions. Perception \& Psychophysics, 5, 113-116.

GiNSBURG, A. P. (1978). Visual information processing based upon spatial filters constrained by biological data (Doctoral dissertation, Cambridge University). Published as AFAMRL-TR-78-129.

Hess, R. F., \& Plant, G. T. (1985). Temporal frequency discrimination in human vision: Evidence for an additional mechanism in the low spatial and high temporal frequency region. Vision Research, 25 , 1493-1500.

HochberG, J. (1971). Perception: Space and movement. In J. A. Kling \& L. A. Riggs (Eds.), Woodworth and Schlosberg's Experimental psychology (pp. 475-550). New York: Holt, Rinehart \& Winston.

JuLESZ, B. (1978). Perceptual limits of texture discrimination and their implications to figure-ground separation. In E. L. J. Leeuwenberg \& H. F. J. M. Buffart (Eds.), Formal theories of visual perception (pp. 205-216). New York: Wiley.

KeLLY, D. H. (1972). Adaptation effects on spatio-temporal sine-wave thresholds. Vision Research, 12, 89-101.

KeLLY, D. H. (1979). Motion and vision: II. Stabilized spatio-temporal threshold surface. Joumal of the Optical Society of America, 69, $1340-1349$.

KLYMENKo, V., WEISSTEIN, N. (1986). Spatial frequency differences can determine figure-ground organization. Journal of Experimental Psychology: Human Perception \& Performance, 12, 324-330.

KoENDERINK, J. J., VAN DoORN, A. J. (1979). Spatiotemporal contrast detection threshold surface is bimodal. Optics Letters, 4, 32-34.

KOFFKA, K. (1935). Principles of Gestalt psychology. New York: Harcourt, Brace.

KuLIKowskI, J. J. (1976). Effective contrast constancy and linearity of contrast sensation. Vision Research, 16, 1419-1431.

Livingstone, M., \& HuBEL, D. (1988). Segregation of form, movement and depth: Anatomy, physiology, and perception. Science, 240, $740-749$.

Meyer, G. E., Dougherty, T. (1987). Effects of flicker-induced depth on chromatic subjective contours. Joumal of Experimental Psychology: Human Perception \& Performance, 13, 355-360.

OlzaK, L. A., \& Thomas, J. P. (1986). Seeing spatial patterns. In K. R. Boff, L. Kaufman, \& J. P. Thomas (Eds.), Handbook of perception and human performance (Vol. 1, pp. 7-1 to 7-56). New York: Wiley.

Ramachandran, V. S., A ANSTis, S. (1986). Figure-ground segregation modulates apparent motion. Vision Research, 26, 1969-1975.

RoBson, D. S. (1959). A simple method for construction of orthogonal polynomials when the independent variable is unevenly spaced. Biometrics, 15, 187-191.

RoBSON, J. (1966). Spatial and temporal contrast sensitivity functions of the visual system. Journal of the Optical Society of America, 56, 1141-1142.

Rubin, E. (1958). Figure and ground. In D. C. Beardslee \& M. Wertheimer (Eds.), Readings in perception (pp. 194-203). Princeton, NJ: Van Nostrand. (Original work published 1921)

Schor, C. M., \& HowarTh, P. A. (1986). Suprathreshold stereo-depth matches as a function of contrast and spatial frequency. Perception, 15, 249-258.

UTTAL, W. R. (1981). A taxonomy of visual processes. Hillsdale, NJ: Erlbaum. 
van Nes, F. L., Koenderink, J. J., \& Bouman, M. A. (1967). Spatiotemporal modulation transfer in the human eye. Journal of the Optical Society of America, 57, 1082-1087.

W Atson, A. B. (1986). Temporal sensitivity. In K. R. Boff, L. Kaufman, \& J. P. Thomas (Eds.), Handbook of perception and human performance (Vol. 1, pp. 6-1 to 6-43). New York: Wiley.

Watson, A. B., \& NaChmias, J. (1977). Patterns of temporal interaction in the detection of gratings. Vision Research, 17, 89-101.

WERTHEIM, A. H. (in press). Visual, vestibular and oculomotor interactions in the perception of object motion during egomotion. In R. Warren and A. H. Wertheim (Eds.), The perception and control of egomotion. Hillsdale, NJ: Erlbaum.

WeISSTEIN, N., \& WoNG, E. (1986). Figure-ground organization and the spatial and temporal responses of the visual system. In E. Schwab $\&$ H. C. Nusbaum (Eds.), Pattern recognition by humans and machines (Vol. 2, pp. 31-63). New York: Academic Press.

Weisstein, N., \& Wong, E. (1987). Figure-ground organization affects the early visual processing of information. In M. A. Arbib \& A. R. Hanson (Eds.), Vision, brain, and cooperative computation (pp. 209-230). Cambridge, MA: MIT Press.

WoNG, E., \& WeIsSTEIN, N. (1982). A new perceptual context superiority effect: Line segments are more visible against a figure than against a ground. Science, 218, 587-589.

Wong, E., \& WeISSTEIN, N. (1983). Sharp targets are detected better against a figure, and blurred targets are detected better against a ground. Joumal of Experimental Psychology: Human Perception \& Performance, 9, 194-202.
Wong, E., \& Weisstein, N. (1984). Flicker induces depth: Spatial and temporal factors in the perceptual segregation of flickering and nonflickering regions in depth. Perception \& Psychophysics, 35, 229-236.

Wong, E., \& Weisstein, N. (1985). A new visual illusion: Flickering fields are localized in a depth plane behind nonflickering fields. Perception, 14, 13-17.

Wong, E., \& Weisstein, N. (1987). The effects of flicker on the perception of figure and ground. Perception \& Psychophysics, 41, $440-448$.

\section{NOTE}

1. In addition, the absolute response times were recorded and analyzed. The absolute response time is the absolute amount of time, in seconds, during which the right region was seen as the background, out of the $30 \mathrm{sec}$ of stimulus presentation (neutral responses were not discounted). The pattern of results for the absolute response times was the same as that reported here for the percent response times. The average neutral times in which the observers did not respond either "right" or "left" was 2.2, 1.5, and $2.0 \mathrm{sec}$, respectively, for Experiments 1, 2, and 3. Most of the neutral time is likely due to the initial reaction time.

(Manuscript received December 23, 1987; revision accepted for publication October 12, 1988.) 